\title{
A INTER-RELAÇÃO ENTRE O PROCESSO DE INTELIGÊNCIA COMPETITIVA E A GESTÃO DA INOVAÇÃO: PROPOSIÇÃO DE UM MODELO TEÓRICO INTEGRATIVO
}

\author{
THE INTERRELATION BETWEEN THE PROCESS OF \\ COMPETITIVE INTELLIGENCE AND THE \\ MANAGEMENT OF INNOVATION: PROPOSITION OF AN \\ INTEGRATIVE THEORETICAL MODEL
}

Thaís Silva

Júlia Mudrik

Frederico Vidigalc

\begin{abstract}
RESUMO
Introdução: Frente às incertezas inerentes ao processo de inovação e às instabilidades dos mercados cada vez mais competitivos, diversos métodos e técnicas tem sido utilizados pelas empresas como apoio ao processo de desenvolvimento de novos produtos e da inovação em si. Apesar das práticas de Inteligência Competitiva (IC) serem reconhecidas como um processo estratégico de apoio à tomada de decisão, poucos trabalhos exploram de forma mais detalhada como os ciclos de IC podem ser incorporados ao processo de gestão da inovação de forma a robustece-lo. Objetivos: Propor um modelo teórico integrativo das práticas de IC com o modelo de inovação Stage-Gate $\AA$. Metodologia: Revisão de literatura. Resultados: Modelo teórico integrativo do Stage-Gate $\AA^{\circledR}$ com os ciclos de IC, no qual um ciclo único está associado às etapas relacionadas ao nível estratégico do processo enquanto ciclos específicos são associados a cada um dos projeto de produto separadamente. Conclusões: A integração dos modelos contribui positivamente para os resultados empresariais ao estimular uma cadeia de agregação de valor em conhecimento que permite a redução contínua das incertezas, bem como maior conhecimento do mercado e dos clientes, dando suporte a tomada de decisão durante todo o processo de inovação. Ressalta-se assim a complementariedade e vantagens do uso combinado das abordagens aqui tratadas.
\end{abstract}

\footnotetext{
a Doutoranda em Administração pela Universidade Federal de Minas Gerais (CEPEAD/FACEUFMG). E-mail: tha.silva25@gmail.com

b Mestre em Administração pela Universidade Federal de Minas Gerais (CEPEAD/FACE-UFMG). E-mail: juliatiso@gmail.com

c Doutor em Ciência da Informação pela Universidade Federal de Minas Gerais (ECI-UFMG). Professor do curso de Administração no grupo IBMEC. E-mail: fvidigal@terra.com.br
} 
Descritores: Gestão da Inovação. Inteligência Competitiva. Tomada de Decisão. Ciclo de Inteligência. Modelo Conceitual.

\section{INTRODUÇÃO}

O sucesso no desenvolvimento, introdução e difusão de novos produtos e serviços para a sociedade é uma atividade-chave para que as empresas se mantenham competitivas e sobrevivam ao longo do tempo. Esse processo, realizado de forma bem sucedida, pode ser entendido como inovação (TIDD; BESSANT; PAVITT, 1997).

De forma geral, as empresas procuram gerar inovações de forma sistêmica e contínua, e para isso devem, prioritariamente, desenvolver a inovação através de um processo formal que contenha as etapas e critérios pelos quais as ideias devem passar (MONTANHA JUNIOR et al., 2008; BAGNO, 2014).

Diferentes autores propõem variadas abordagens para a estruturação e formalização do processo de inovação (TIDD; BESSANT; PAVITT, 1997; COOPER, 1993; CLARK; WHEELWRIGHT, 1993). Além disso, diversos métodos e técnicas são comumente utilizados para apoiar o processo de inovação e desenvolvimento de novos produtos, sendo aplicados como forma de reduzir as incertezas e estruturar o processo de tomada de decisão. Oliveira e Rozenfeld (2009) citam, por exemplo, o Planejamento de Cenários, o Technology Roadmapping (TRM) (PHAAL; FARRUKH; PROBERT, 2004), a Análise SWOT, TRIZ, Balance Scored Card (BSC), Inteligência Competitiva (IC), entre outros.

Especificamente em relação à utilização da IC, essa pode ser compreendida como um conjunto de atividades para processamento, análise e disseminação de informação que dão subsídios para a tomada de decisão estratégica (PEREIRA; CARVALHO; JORDÃO, 2016). Tais atividades, nos últimos tempos, se tornaram mais frequente devido principalmente às mudanças estruturais dos processos internos e da dinâmica de funcionamento sofrida pelas empresas. Essas transformações, advindas de alterações nos ambientes de negócio, deram relevância aos processos de IC que passaram a possibilitar o acompanhamento, interpretação e respostas das empresas às modificações 
sofridas.

Já em relação ao uso de IC no processo de inovação, autores comprovaram empiricamente (TANEV; BAILETTI, 2008) e através de revisão da literatura (TANEV; BAILETTI, 2008; HUSSEIN; FARZANEH; FARHAM, 2011) a relação positiva do uso de informações de IC e a performance de inovação das empresas. Apesar disso, são poucos os trabalhos encontrados na literatura que exploram de forma mais detalhada a maneira como os dois processos (i.e. Inovação e Inteligência Competitiva) podem ser unificados visando melhorar o desempenho geral competitivo das empresas (CANONGIA; SANTOS; ZACKIEWICZ, 2004; PANIZZON; OLIVEIRA, 2013).

Assim sendo, diante do exposto, o objetivo desse artigo foi, a partir de uma revisão da literatura, desenvolver um modelo conceitual que identifique as maneiras com que o ciclo de inteligência competitiva e suas ferramentas podem e devem ser utilizados em conjunto com os modelos de inovação. Essa sistematização é relevante ao unificar os benefícios já levantados pela literatura tanto de inovação como de IC, e ao potencializar e reforçar como as sinergias entre os dois processos podem estimular uma cadeia de agregação de valor em conhecimento e influenciar a vantagem competitiva das empresas.

Para fins desse trabalho utilizou-se o modelo de inovação Stage-Gate® proposto por Cooper (1993), por ser considerado na literatura como um dos mais utilizado pelas empresas (LONGANEZI; COUTINHO; BOMTEMPO, 2008; SILVA; BAGNO; SALERNO, 2014).

\section{REFERENCIAL TEÓRICO}

\subsection{Inteligência Competitiva}

O conceito de Inteligência Competitiva (IC), embora esteja integrado a muitas práticas organizacionais de empresas líderes de mercado, remete a épocas militares (ADIDAM; BANERJEE; SHUKLA, 2012). Podendo ser rastreado até mais de 5.000 anos de história chinesa, textos de IC mencionam a obra de Sun Tzu, A Arte da Guerra, como um texto seminal que oferece uma descrição de como desenvolver a inteligência para aplicações militares. A indústria de chá 
britânico, por exemplo, também tem suas raízes em IC, já em 1965 enviou trabalhadores à China para reunir informações sobre a importância do chá e seu potencial para contribuir para a economia britânica (CALOF; WRIGHT, 2008).

Como aponta Mohsin, Halim e Ahmad (2015) para compreender o que é IC é preciso conceituar primeiro inteligência. O conceito de Inteligência está ligado aos benefícios coletivos de valor agregado obtidos a partir de ativos intangíveis, como exemplo, o conhecimento dos funcionários, da gestão, dos stakeholders e clientes. Conhecimento e experiência estariam assim atrelados. É importante ressaltar que embora a informação seja o cerne da IC, este último almeja objetivos maiores do que somente a recolha de dados, ou seja, não é, como corroboram Rouach e Santi (2001), um processo de gestão da informação.

Inteligência Competitiva, segundo Calof e Skinner (1998), é uma atividade sistemática que envolve recomendações de ações sobre o ambiente externo que afetam uma situação competitiva de uma empresa ou país. Tais recomendações são decorrentes do que é conhecido como ciclo de IC que, para fins desse trabalho, considerou-se o proposto por Amaral, Garcia e Aliprandini (2008) que é composto por seis fases: (i) identificação das necessidades de informação; (ii) planejamento; (iii) coleta das informações; (iv) análise; (v) disseminação e (vi) avaliação. É importante ressaltar que se encontra na literatura divergências quanto ao número de etapas constitutivas do ciclo (GOMES; BRAGA, 2004; MILLER, 2002), contudo, como expõe Pizzol, Todesco e Todesco (2016) as fases de coleta, análise e disseminação parecem ser comuns à maioria dos autores.

Nesse sentido, a primeira fase corresponde à identificação das necessidades de informação e gerará, como aponta Pereira, Carvalho e Jordão (2016), os "Tópicos chave" de inteligência, também conhecido pelo processo Key Intelligence Topics (KIT) que foi elaborado por Herring em 1999. De acordo com o KIT, existem três categorias de informação. A primeira categoria são as informações que suprem as necessidades estratégicas e táticas da organização que advém de rotinas de tomada de decisão e planejamento que são repetidas com frequência dentro do processo de trabalho de uma empresa. Já na segunda categoria, as informações são voltadas para a identificação e descrição de atores 
chave do ambiente de negócio, como competidores, fornecedores, clientes e parceiros através da elaboração de perfis que eventualmente melhorem futuras relações (PEREIRA; CARVALHO; JORDÃO, 2016). Sobre esse aspecto Adidam, Banerjee e Shukla (2012) expõe que, em alguns casos, a melhor inteligência é obtida através de contatos humanos informais decorrentes de tais atores chave.

Por fim, a última categoria de informação são aquelas voltadas para o monitoramento ambiental que visam levantar informações que permitam à organização se antecipar a eventos críticos do mercado, ou mesmo compreender melhor acontecimentos e fatos já ocorridos (PEREIRA; CARVALHO; JORDÃO, 2016). Normalmente se busca informações sobre o ambiente político, econômico, social, tecnológico, ecológico e legal.

Após a identificação de necessidade de informações se inicia a segunda fase do ciclo, o planejamento, que determinará a forma como toda a atividade de inteligência competitiva será desenvolvida em termos de missão, objetivo, estratégias e ações que serão tomadas. Já na terceira fase (i.e. coleta de informações) serão realizadas as etapas de busca, adequação e processamento das informações úteis à resolução dos KITs (PIZZOL; TODESCO; TODESCO, 2016; PEREIRA; CARVALHO; JORDÃO, 2016).

Com a explosão de informações disponíveis publicamente, que vem ocorrendo nos últimos tempos através de blogs, redes sociais e outros meios de comunicação eletrônico, as fontes de informação para a IC se expandiram (BOSE, 2008). Todavia, ao mesmo tempo em que a Internet facilita o acesso a dados sobre o mercado competitivo e sobre os desejos e avaliações dos clientes atuais e potenciais, ela também cria desafios de assimilação da informação (MENON et al., 1999). Tal desafio exige que as empresas otimizem seus sistemas de inteligência.

Ao que se refere à quarta fase do ciclo, análise das informações, os dados e informações coletados são transformados em inteligência. Aqui se pode fazer classificações quanto ao tempo de vida útil, ao público a que se dedica, e aos processos e fontes utilizados, por exemplo. Na quinta fase, disseminação da inteligência, as informações de inteligência são expostas e as alternativas de 
ação que guiarão a tomada de decisão são apresentadas. Por fim, a sexta fase do ciclo é a avaliação, que corresponde ao acompanhamento e avaliação do que foi planejado bem como se a IC está sendo utilizada de maneira estratégica no processo de tomada de decisão (PIZZOL; TODESCO; TODESCO, 2016; PEREIRA; CARVALHO; JORDÃO, 2016).

Rouach e Santi (2001) expõem que as atividades de IC de um modo geral devem ser legais e respeitar os códigos de ética, não podendo ser confundido com o que é conhecido como espionagem. Nesse sentido, deve tratar-se de uma transferência de conhecimento do ambiente para a organização dentro de regras pré-estabelecidas (ROUACH; SANTI, 2001). Pode ser assim compreendida como um radar, como destacam os autores supracitados, que detecta novas oportunidades ou ajuda a evitar desastres, alertando as empresas para a importância de se observar o ambiente ao qual estão inseridas.

Devido ao seu impacto nas decisões de negócio e ao seu poder de moldar as decisões estratégicas de determinada empresa, as atividades de IC devem ser, de acordo com Priporas, Gatsoris e Zacharis (2005), de responsabilidades da gestão de topo, mas em mercados desenvolvidos também pode ser utilizada por níveis táticos e operacionais.

Inicialmente tida como uma atividade especializada contida dentro da pesquisa de marketing, a IC evoluiu e se desenvolveu como um campo distinto e as atividades desenvolvidas têm vindo a servir a todas as funções de negócio (ADIDAM; BANERJEE; SHUKLA, 2012).

$\mathrm{Na}$ visão de Priporas, Gatsoris e Zacharis (2005), IC pode ser um processo ou um produto. Como um processo, tem vários passos a serem seguidos incluindo: planejamento, coleta, análise, disseminação organizacional para os tomadores de decisão, ou seja, passos que fazem parte de um ciclo de IC (KAHANER, 1996; MILLER, 2002). Da mesma maneira, IC é considerada um produto quando a inteligência produzida assessora os tomadores de decisão na elaboração de uma estratégia ou ao fazer uma escolha.

De uma maneira geral, um sistema de IC contínuo pode trazer outros benefícios além das vantagens financeiras e de desempenho da empresa (ADIDAM; BANERJEE; SHUKLA, 2012). Ao proporcionar a concepção de perfis 
de informação, que mais adiante podem servir como meio de comparação, os sistemas de IC ajudam a identificar padrões de pontos fortes, fracos, estratégias, objetivos, posicionamento de mercado e provável reação dos concorrentes (BOSE, 2008). Além disso, também há contribuições para o desenvolvimento de novos produtos e na penetração de mercado.

\subsection{Modelos de Gestão da Inovação}

A primeira definição de inovação foi proposta por Schumpeter (1934). Ele a associou ao desenvolvimento econômico e a definiu como uma nova combinação de recursos produtivos. Seu trabalho definiu cinco casos específicos de inovação: introdução de novos produtos, novos métodos de produção, exploração de novos mercados, conquista de novas fontes de suprimento e novas formas de organização de negócios. Desde então, o conceito de inovação evoluiu significativamente e atualmente ela não é mais concebida como um resultado específico de ações individuais, mas como o resultado de um processo.

Baregheh, Rowley e Sambrook (2009) definem a inovação como um processo multi estágio através do qual as organizações transformam ideias em bens, serviços ou processos novos ou significativamente melhorados com 0 objetivo de progredir, competir ou diferenciarem-se com sucesso no mercado.

$\mathrm{Na}$ literatura de gestão da inovação existem diferentes modelos voltados para a estruturação do processo com foco em diferentes dimensões tecnológica, organizacional, processos e produtos (LOPES et al., 2012; SILVA; BAGNO; SALERNO, 2014). De modo geral, os modelos mais reconhecidos, concentram-se na atividade de desenvolvimento de produtos e foram desenvolvidos através de estudos em empresas de grande porte, com atividades de pesquisa e desenvolvimento bem estruturadas e recursos previstos em orçamento formal (BAGNO, 2014). Como exemplos reconhecidos, podemos citar o Funil de desenvolvimento (CLARK; WHEELWRIGHT,1992), o Stage-Gate® de Cooper (1993), e o Funil de inovação aberta de Docherty (2006), que inclui conceitos relacionados à busca de fontes externas de tecnologia e inovação (open inovation). A etapa de geração de ideias é um ponto comum de início da 
maioria dos modelos de inovação, e está diretamente ligada às oportunidades provindas do desenvolvimento tecnológico e demandas identificadas do mercado (ou ambos de forma combinada) (SILVA; BAGNO; SALERNO, 2014).

O modelo de Stage-Gate ${ }^{\circledR}$ de Cooper (1993) é um dos modelos mais influentes na literatura (LONGANEZI; COUTINHO; BOMTEMPO, 2008; SILVA; BAGNO; SALERNO, 2014) e por isso foi escolhido como modelo base para este estudo.

O Stage-Gate ${ }^{\circledR}$ é um processo sequencial de cinco etapas (Stages) e decisões (Gates) que tem como principal objetivo levar ideias de novos produtos ao lançamento no mercado. Segundo Cooper (2003), cada Stage, no qual o time de projeto obtém as informações necessárias e realiza a subsequente integração e análise de dados, é seguida por um Gate, onde decisões de prosseguir ou parar (Go/Kill) para cada projeto são tomadas. O modelo completo é apresentado na figura 1.

Figura 1- Modelo Stage-Gate $\AA$

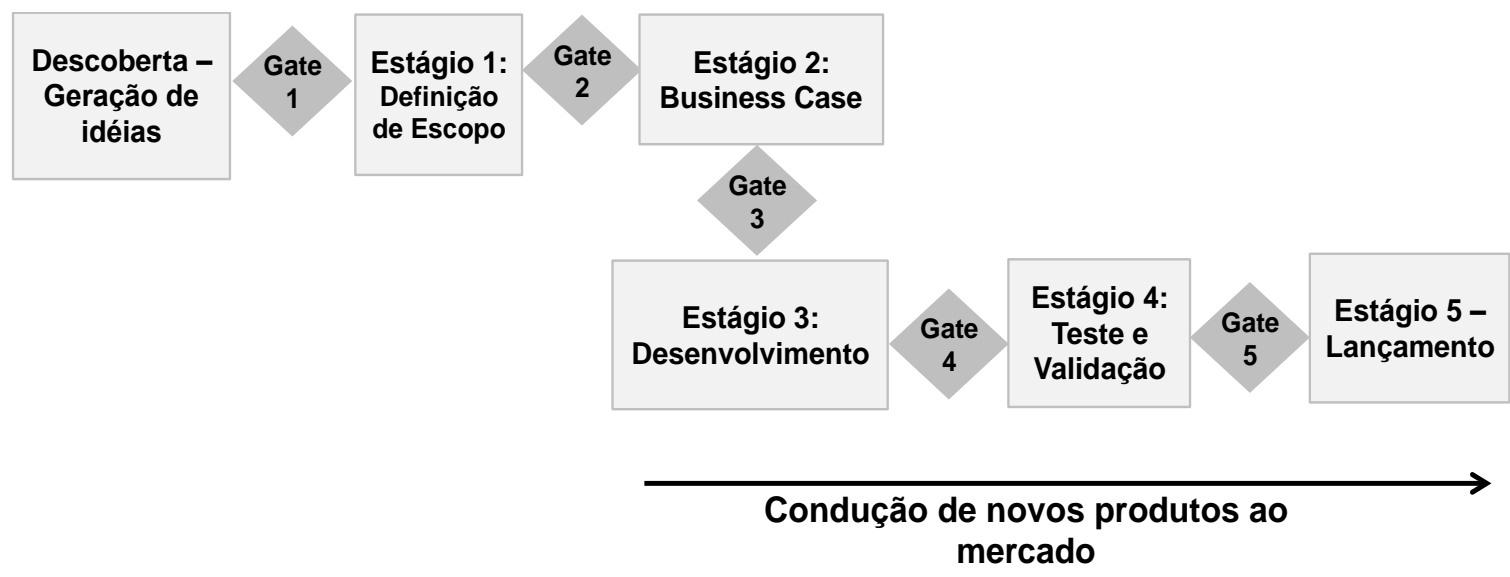

Fonte: Adaptado de Cooper, 1993.

A primeira atividade do Stage-Gate ${ }^{\circledR}$ corresponde a um estágio preliminar, cujo objetivo é auxiliar o processo de geração de ideias através do brainstorming, por exemplo, seguida do Gate cujo objetivo é a seleção inicial das ideias geradas para terem continuidade no Processo de Desenvolvimento de Produtos (PDP). Uma ideia, materializada em um produto, pode ser vista como alternativas tecnológicas identificadas para atender a expectativas da demanda em determinado mercado (GARCEZ et al., 2005). As ideias podem vir de 
diferentes fontes, incluindo fontes internas a organização (ex. Funcionários), e externas, como análise de mercado, clientes, fornecedores, entre outros.

O estágio 1 compreende uma investigação preliminar para a definição do escopo dos produtos a partir das ideias selecionadas. Segundo Garcez et al. (2005), nesta fase são conhecidas as características gerais do mercado e seus possíveis segmentos, podem ser formulados possíveis cenários simplificados para os novos produtos, além de se iniciar a avaliação de viabilidade técnica, investimento e prazo de desenvolvimento, mas apenas com informações qualitativas.

No estágio 2 o escopo dos projetos dos produtos, tanto técnico quanto comercial, são especificados na forma de um Bussiness Case que inclui a definição do produto, o plano de desenvolvimento e a justificativa do projeto.

Estes dois primeiros estágios, somados ao estágio preliminar, correspondem ao que Cooper (1988) chama de pré-desenvolvimento. Esta etapa está no nível estratégico das empresas e antecede o início do desenvolvimento dos produtos (nível projeto). É no pré-desenvolvimento que ocorre a definição do portfólio de produtos com base no plano estratégico da empresa, nas restrições de recursos, informações sobre consumidores, e tendências tecnológicas e de mercado (ROZENFELD, 2006).

O estágio 3, denominado por Cooper (1993) como Desenvolvimento, prevê o desenvolvimento técnico do produto no nível de projeto, incluindo seus processos de produção e operação. O resultado da etapa é um protótipo ou amostra do produto, e também um plano de marketing mais completo e formal, com a definição do mercado-alvo, estratégia e posicionamento do produto e o marketing mix (preço, distribuição, publicidade e propaganda, estratégia da força de vendas, serviços acoplados) (GARCEZ et al., 2005).

Já no estágio 4, Validação e Testes, são realizados tanto testes técnicos (em laboratório ou em painéis com usuários, por exemplo) e comerciais (comercialização em uma região, por exemplo), buscando a aprovação tanto técnica quanto do conceito comercial do novo produto. Busca-se identificar falhas técnicas, de design e do próprio plano de marketing do produto para que 
sejam realizadas modificações visando aumentar as chances de sucesso no mercado.

Por fim, o estágio 5 representa o lançamento comercial do produtos, incluindo as atividades de propaganda, distribuição em massa e comercialização.

Cada estágio é desenhado para levantar as informações necessárias para reduzir as incertezas e riscos dos projetos, sendo assim, os requisitos de informação definem o propósito de cada estágio do processo. Cooper (1993) também destaca que o custo do estágio vai aumentando e o nível de incerteza reduzindo a medida em que o processo vai sendo realizado, ou seja, os custos de execução e de mudança associados aos estágios finais são superiores aos iniciais.Por esse motivo, o autor encoraja que as mudanças aconteçam o quanto antes no processo, uma vez que raramente um produto mantem seu conceito original do início ao fim do PDP.

Apesar de existirem críticas à linearidade e possível engessamento gerado pelos diferentes modelos que visam estruturar o processo de inovação das empresas, os autores argumentam por uma convergência em torno de uma estrutura básica e afirmam que é necessário um adequado equilíbrio entre simplificações e representações (BAGNO, 2014).

\section{INOVAÇÃO E INTELIGÊNCIA COMPETITIVA}

A importância da inovação para se alcançar a competitividade é crescente, entretanto as estratégias adotadas pelas empresas, como apontam Canongia, Santos e Zackiewicz (2004), enfrentam desafios inerente ao processo de inovação como, por exemplo, a incerteza, a capacidade de análise de rotas alternativas, a mobilização de competências, a valorização da criatividade, dentre outras.

De certo, é possível perceber nas práticas de IC elementos que favorecem o sucesso perante tais desafios que, em muitos casos, podem estar relacionadas à inovação com que uma empresa deseja alcançar. Desse modo, a transposição dos mesmos é fator crucial para que se possa introduzir com sucesso novos produtos, processos e serviços (TANEV; BAILETTI, 2008). Como salientam 
Lemos e Porto (1998), a utilização da informação em atividades de inteligência competitiva pode melhorar os modelos de inovação já que, segundo os autores, usar somente o avanço tecnológico como instrumento de competitividade já não é novidade.

Especificamente em relação ao modelo de inovação tratado nesse artigo, o Stage-Gate $\AA$, e ao ciclo de IC, algumas convergências podem ser observadas, o que justifica a utilização de um modelo que integre tais abordagens. Algumas delas são: o suporte a criação de inovação na empresa; os resultados de cada fase dos processos objetivam fomentar a tomada de decisão; ambos obtêm informação, dados e os transformam em conhecimento; podem ser usados de maneira flexível.

A figura 2, a seguir, apresenta o modelo de integração proposto neste artigo, ressaltando que além de convergentes, as abordagens podem se complementar, acarretando vantagens na utilização combinada. Essas vantagens serão detalhadas ao longo do tópico.

Figura 2 - Modelo conceitual de integração Stage-Gate ${ }^{\circledR}$ e ciclo de IC

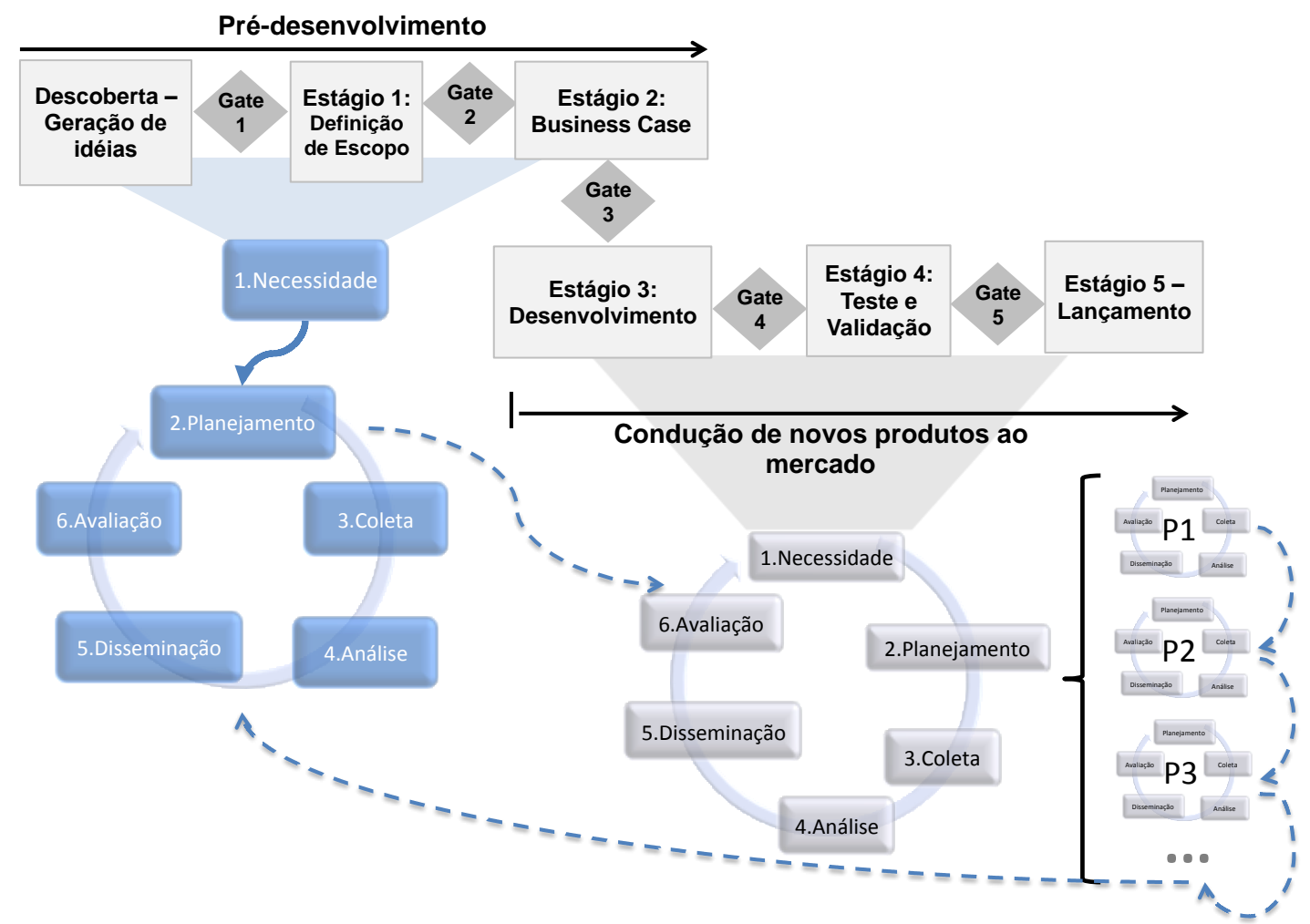

Fonte: Elaborado pelos autores. 
Como evidenciado na figura 2, a inteligência competitiva deve atuar em todos os estágios do modelo de inovação. Entretanto, vale salientar que as ferramentas utilizadas e os produtos decorrentes da IC se alteram na medida em que a empresa conclui e avança nas etapas do Stage-Gate®, principalmente devido às mudanças de objetivos.

A fase de pré-desenvolvimento (etapa de descoberta, estágio 1 e 2) está no nível estratégico da organização e seu objetivo é definir o portfólio de produtos que será desenvolvido nos estágios seguintes. Para esta etapa é previsto um ciclo único de IC, integrado para todos os projetos de produtos em análise, em que há maior destaque para informações da estratégia da empresa e análise mais globais do mercado externo e recursos internos. A partir do estágio 3 Desenvolvimento, as decisões estão no nível tático e operacional e, em sua maior parte, são tomadas para cada projeto separadamente. Nesse sentido, são previstos ciclos de IC específicos por projeto (identificados na figura como P1, P2, P3) que estão relacionados entre si e que retroalimentam o ciclo da etapa de pré-desenvolvimento.

A fase de pré-desenvolvimento é o momento em que a empresa analisa - ambiente externo e interno para descobrir tendências, monitorar acontecimentos e analisar seus recursos e tecnologias na busca de novas oportunidades. Visto que ainda não se sabe qual são as melhores alternativas de produto e suas implicações mercadológicas e técnicas, nesse estágio as incertezas são maiores, o que faz com que o uso da IC se torne mais crítico e essencial.

O tipo de necessidade de informação nas 3 etapas da fase de prédesenvolvimento é sempre o mesmo, o que não quer dizer que o ciclo de IC seja estático. Essa característica está indicada na figura pela separação da fase "Necessidade" do restante do ciclo, pois uma vez que ela tenha sido bem executada levando em conta as necessidades do processo de inovação, nos próximos ciclos poderão acontecer apenas revisões quando necessário. $O$ restante do ciclo (fases 2 a 6 ) roda normalmente e continuamente. O que faz com que o ciclo de inteligência seja importante são as ferramentas utilizadas e a maneira como a informação é transformada em produto de inteligência e utilizada 
na tomada de decisão em relação ao portfólio de projeto que será posteriormente desenvolvido. Já que nesses estágios inicias os desembolsos financeiros são reduzidos em comparação aos valores despendidos em etapas subsequentes do desenvolvimento do produto, a tomada de decisão e detalhamentos obtidos aqui podem determinar e/ou evitar possíveis ameaças e prejuízos.

De acordo com Marcial e Costa (2001), é muito importante que as empresas em fase de monitoramento do ambiente tracem cenários prospectivos. Esses, segundo os mesmos autores, são entendidos como uma descrição coerente de uma situação futura e de como se dão os direcionamentos da situação presente para a direção futura. Como fonte de diminuição de incertezas e apoio a tomada de decisão, a utilização de prospecção de cenários nessa fase de pré-desenvolvimento pode ser determinante. A inteligência competitiva serve então como base, principalmente se pensarmos nas fases de coleta e análise de informações, que compõem o ciclo e que podem subsidiar e melhorar a qualidade dos cenários traçados (MARCIAL; COSTA, 2001).

Vale salientar que na análise do ambiente externo, um tipo especifica de ferramenta de IC pode ser utilizado. Denominada análise PEST (política, econômica, social-cultural e tecnológica) ou de maneira mais abrangente análise PESTEL (adição da esfera ecológica e legal), a ferramenta proporciona uma visão de fatores que podem causar impactos positivos ou negativos no negócio da empresa, como exemplo, as intervenções governamentais (CASTOR, 2000; MACHADO; SOUZA; VALVERDE, 2010). Os autores mencionados anteriormente expõem que é importante que essa análise seja combinada com outras ferramentas e análises, como por exemplo, as Cinco Forças de Porter, e que esteja claro quais os objetivos se está buscando para que o excesso de informação não seja um fator negativo.

Como corroboram Panizzon e Oliveira (2013), quando se está gerindo oportunidades, a IC se desenvolve na formação de equipes especializadas, grupos de futuro, ou vigilância que constroem rotinas de observação, com o uso ou não de redes (Open Innovation), na busca de sinais ambientais e padrões nos diversos cenários. Na mesma perspectiva, Franco e Barbeira (2008) complementam ainda que o melhor sistema de inteligência competitiva é aquele 
que proporciona a avaliação, a validade e confiabilidade da informação, bem como possibilita sua interpretação e organização de forma apropriada a proporcionar maior detalhamento de características do mercado, tendências, postura dos concorrentes e segmentos de clientes, por exemplo. Ter essas informações detalhadas dá suporte à tomada de decisão sobre em quais projetos a empresa deve investir.

Para o melhor desenvolvimento especialmente do estágio 2, no qual é especificado o escopo do projeto tanto comercial como técnico, é interessante que a IC gere produtos relativos também ao ambiente interno. Esses produtos podem permitir que a empresa faça uma gestão mais estratégica do conhecimento que ela própria produz e que pode advir da base de dados da própria empresa ou do conhecimento disseminado pela proximidade entre as pessoas (FRANCO; BERBEIRA, 2008). As informações podem se referir, por exemplo, a como ocorreu o desenvolvimento de projetos semelhantes em termos técnicos e de respostas obtidas dos clientes e concorrentes ou quais competências ainda são necessárias para que determinado projeto novo possa ser executado.

Quando se chega ao desenvolvimento do produto, estágio 3, um novo ciclo de IC se inicia, já que nesse momento os esforços, tanto financeiros como operacionais, da empresa são canalizados para determinados projetos, previamente escolhidos com o auxílio dos produtos de inteligência gerados nas etapas anteriores. Neste estágio o processo de IC começa a atuar já em níveis táticos e específicos para cada projeto de produto, e os produtos gerados através da IC têm auxilio ainda maior nas tomadas de decisão.

Como acontece um ciclo para cada novo produto, diferentemente da etapa de pré-desenvolvimento, o ciclo é sempre completo, incluindo a fase $1 \mathrm{de}$ definição de necessidade de informação. Essa diferença se dá uma vez que o foco neste momento é no projeto de um produto específico, no qual as informações devem deixar de ser mais amplas para serem específicas ao segmento de mercado e tecnologias do produto, que podem divergir entre os projetos. 
Vale salientar a importância da formação de redes intraorganizacionais nesse estágio, e também nos que o sucedem, principalmente para que se mantenha um código único de informação em relação ao que já foi identificado no ciclo desenvolvido anteriormente (CUNHA; MEDEIROS, 2007; RODRIGUES et al., 2009). Além disso, como expõe Cunha e Medeiros (2007), as empresas nesse estágio podem atuar também em redes interorganizacionais para usufruir de seus vínculos externos que compensariam habilidades ou capacidades que não possuem, tornando-as mais competitivas e robustas.

Nesse sentido, destaca-se a importância da fase de identificação da necessidade de informação, presente no ciclo de IC, na qual, como já elucidado, são traçados, através do processo de KIT's, os perfis de atores chaves do negócio que poderão ser posteriormente parceiros dentro dessa atuação em rede.

Como destaca Morais e Luz (2010), em uma sociedade do conhecimento, que exige que as empresas aperfeiçoem cada vez mais seus sistemas de inteligência, e na qual a competitividade e a informação são peças essenciais, a cooperação, em diversos níveis, se torna um diferencial competitivo para os atores, podendo colaborar assim com o aumento da produtividade e a diminuição dos custos. As redes de cooperação são, desse modo, uma causa e uma consequência da natureza evolutiva das formas de concorrência dos mercados (BORTOLASO; VERSCHOORE; ANTUNES JÚNIOR, 2013).

Além das vantagens competitivas advindas do contato intra e interorganizacional proporcionado pela formação de redes se encontram na literatura suporte para importância de aumentar ainda mais a conectividade com o objetivo de alavancar o processo de inovação (NASCIMENTO; LUFT; FREITAS, 2012). Especificamente em relação ao desenvolvimento de produtos, o uso da inteligência competitiva através de parcerias com os clientes é ressaltado. Nesse âmbito o cliente é entendido como prosumer, ou seja, é ao mesmo tempo produtor e consumidor de conteúdo (NASCIMENTO; HEBER; LUFT, 2012; VIDIGAL; MARQUES, 2016).

Semelhante ao que é conhecido como co-criação, o termo crowdsourcing se refere a esse modelo de produção e desenvolvimento que utiliza os 
conhecimentos coletivos e voluntários disponíveis na internet pelos prosumers para resolver problemas, criar soluções e conteúdos ou desenvolver novos produtos e tecnologias (NASCIMENTO; LUFT; FREITAS, 2012).

Desta maneira, ao iniciar no estágio 3 o desenvolvimento do produto, os prosumers podem contribuir, de acordo com Kuipers (2012) através de feedbacks abundantes e constantes, através de comunidades formadas em torno da marca ou focados em uma ideia e no teste de novos conceitos antes de lançá-los efetivamente ao mercado, processo que será de fundamental importância no desenvolvimento do estágio 4.

Em relação às comunidades formadas ao redor de marcas e ideias, essas podem fornecer as empresas importantes insights sobre intenção de compra, recompra e recomendação, que podem determinar os caminhos que serão seguidos no desenvolvimento do produto (ALMEIDA et al., 2011). Como corrobora Ferreira (2014), as informações que são emitidas e coletadas na rede de consumidores, se bem analisadas e canalizadas, são fontes preciosas e diferenciadoras para as organizações que as utilizam.

Assim, de uma maneira geral, o produto de inteligência gerado pelos prosumers, bem como aquele fornecido através das redes inter $e$ intraorganizacionais e da análise PESTEL do ambiente fornecem em conjunto, nesse estágio 3 , as indicações do melhor marketing mix a ser desenvolvido, bem como o estabelecimento de um mercado alvo e estratégias de posicionamento. Destaca-se que muitos dos produtos de IC utilizados nesse estágio, como já mencionado, decorrem do ciclo anteriormente ocorrido no pré-desenvolvimento.

Já em relação ao estágio 4, validação e teste, os produtos para a tomada de decisão podem ser aperfeiçoados em decorrência principalmente do contato com prosumers, como já mencionado. Aqui se salienta a contribuição da utilização dos conhecidos influenciadores digitais, que são pessoas que conseguem influenciar sua audiência, usando da reputação adquirida através de blogs, sites ou redes sociais, a praticar uma determinada ação como comprar um produto, experimentá-lo ou até mesmo testá-lo (SANTOS; SILVA; SANTOS, 2016). Nesse sentido, além de contribuições que poderiam ser obtidas para 0 melhoramento do produto final, relacionadas às falhas técnicas e de design, 
subsídios também poderiam ser captados em relação à consolidação do plano de marketing estabelecido, bem como indícios do sucesso do produto no mercado.

Ademais, a utilização de redes inter organizacionais pode, mais uma vez, ser útil, agora especificamente no estágio referido. Quando se trabalha em conjunto, por exemplo, com fornecedores, a partir do estabelecimento prévio de seus perfis, pode-se acordar sistemas de qualidade que facilitariam o processo de teste técnico em laboratórios de produto (CÔRTES; COUTINHO; CASTANHA, 2002). Essa parceria poderia proporcionar a empresa vantagem competitiva, por exemplo, ao realizar mais rapidamente, mas de maneira eficiente e confiável, os processos de validação e teste, passando assim para o estágio 5, lançamento do produto, de maneira mais rápida que seus concorrentes.

De fato, os perfis dos fornecedores, captados através do radar de IC, poderiam detalhar informações que sejam úteis em todos os estágios aqui analisados, determinando, de maneira segura, que a transição de um estágio para o seguinte aconteça de maneira a evitar retrabalhos e promovendo economias de custo.

Por fim, o estágio 5, lançamento do produto, todos os produtos de IC gerados aos longo dos 4 estágios anteriores são efetivados no produto e em seu lançamento no mercado. As comunidades de marca e influenciadores digitais, por exemplo, são boas ferramentas que também podem ser utilizadas nesse estágio.

É importante que o ciclo seja constantemente avaliado quanto a sua eficiência e eficácia, e que ele aconteça de maneira continuada para que informações coletadas pós-lançamento sejam utilizadas como "fonte" de um novo ciclo, evidenciada pelas linhas pontilhadas na figura. Como enfatizam Panizzon e Oliveira (2013) a partir do momento que a empresa já construiu uma base de conhecimento, ao mesmo tempo em que isso possibilita o acesso a novas informações do mercado e do cliente, fundamenta o suporte e 0 planejamento de inovações incrementais, o que iniciaria novamente o modelo integrado. 


\section{CONSIDERAÇÕES FINAIS}

O mercado está em constante mudança e cada vez mais globalizado, o que amplia a concorrência. Além disso, a complexidade tecnológica está aumentando e os ciclos de vida dos produtos estão encurtando. Todas estas características ressaltam a inovação contínua como um processo essencial para a competitividade e sobrevivência das empresas e altamente dependente da análise e tratamento de informações para gerar conhecimento.

Nesse panorama, os produtos gerados em decorrência de um processo de inteligência competitiva ganharam destaque, como um meio de ganhar habilidade e rapidez para mudar, bem como de adquirir disposição para adotar novas tecnologias e procedimentos de trabalho, convertendo assim conhecimento em produtos e ações que provoquem diferencial perante 0 mercado (MARCO, 1999).

Com o propósito de ressaltar a importância e contribuição das atividades de IC no processo de inovação, objetivou-se nesse estudo propor um modelo conceitual integrado do modelo de inovação Stage-Gate ${ }^{\circledR}$ com o ciclo de IC, focando em destacar suas complementaridades. O modelo se justifica e se torna importante ao partir-se da premissa de que métodos e técnicas devem ser utilizados para apoiar o processo de inovação e desenvolvimento de novos produtos, sendo aplicados como forma de reduzir as incertezas e estruturar 0 processo de tomada de decisão. Além disso, Cooper (1993) evidencia que a utilização de modelos de inovação não devem ser entendidos somente como um sistema de entrada de dados. Longanezi, Coutinho e Bomtempo (2008), corroboram com essa perspectiva ao explanarem que não é raro o equívoco nas organizações de utilizarem o modelo como uma solução para inovação. Dessa maneira, para um sistema inovador eficaz é preciso que as informação criem significado e construam conhecimento capaz de robustecer o processo de inovação.

Para estudos futuros sugere-se a aplicação prática do modelo proposto. Sugere-se também o foco em algum estágio do modelo integrativo para que a complementaridade prática possa ser melhor verificada. 


\section{REFERÊNCIAS}

ADIDAM, P. T.; BANERJEE, M.; SHUKLA, P. Competitive intelligence and firm's performance in emerging markets: an exploratory study in India. Journal of Business \& Industrial Marketing, v. 27, n. 3, p. 242-254, 2012.

ALMEIDA, S. O. de et al. Os Efeitos da Participação em Comunidades Virtuais de Marca no Comportamento do Consumidor: Proposição e Teste de um Modelo Teórico. RAC: Revista de Administração Contemporânea, Curitiba, v. 15, n. 3, p. 366-391, maio/jun. 2011.

AMARAL, R. M.; GARCIA, L. G.; ALIPRANDINI, D. H. Mapeamento e gestão de competências em inteligência competitiva. Ciência da Informação, v. 37, n. 2, mar./ago. 2008.

BAGNO, R. B. Inovação como uma nova função organizacional: caracterização a partir da experiência de empresas industriais de grande porte no Brasil. 2014. 199 f. Tese (Doutorado em Engenharia de Produção) Universidade de São Paulo, Departamento de Engenharia de Produção, São Paulo, 2014.

BAREGHEH, A.; ROWLEY, J.; SAMBROOK, S. Towards a multidisciplinary definition of innovation. Management Decision, v. 47, n. 8, p. 1323-1339, 2009.

BORTOLASO, I. V.; VERSCHOORE, J. R.; ANTUNES JÚNIOR, J. A. V.

Práticas de Gestão de Redes de Cooperação Horizontais: Proposição de um Modelo de Análise. Contabilidade, Gestão e Governança, Brasília, v. 16, n. 3 , p. 3-16, set./dez. 2013.

BOSE, R. Competitive intelligence process and tools for intelligence analysis. Industrial Management \& Data Systems, v. 108, n. 4, p. 510-528, 2008.

CALOF, J. L.; WRIGHT, S. Competitive intelligence a practitioner, academic and inter-disciplinary perspective. European Journal of Marketing, v. 42, n. 7, p. 717-730, 2008.

CALOF, J. L.; SKINNER, W. Competitive intelligence for managers: a brave new world. Optimum, v. 28, p. 38-43, 1998.

CANONGIA, C.; SANTOS, D. M. S. M. M.; ZACKIEWICZ, M. Foresight, inteligência competitiva e Gestão do conhecimento: instrumentos para a Gestão da inovação. Gestão \& Produção, v. 11, n. 2, p. 231-238, maio/ago. 2004.

CASTOR, B. V. J. Planejamento Estratégico em Condições de Elevada Instabilidade. Revista da FAE, Curitiba, v. 3, n. 2, p. 1-7, maio/ago. 2000. 
CLARK, K. B.; WHEELWRIGHT, S. C. Managing new product and process development: text and cases. New York: The Free Press, 1993.

CLARK, K. B.; WHEELWRIGHT, S. C. Revolutionizing product development: quantum leaps in speed, efficiency, and quality. New York: Simon and Schuster, 1992.

COOPER, R. G. Predevelopment activities determine new product success. Industrial Marketing Management, v. 17, n. 3, p. 237-247, 1988.

Winning at new products: accelerating the process from idea to launch. Massachusetts: 1993.

. Profitable product innovation: the critical success factors. In: SHAVININA, L. V. The international handbook on innovation. 2003. p. 139-157.

CÔRTES, A. C. F.; COUTINHO, I. E.; CASTANHA, A. B. Sistemas de gestão de qualidade e gestão ambiental: um estudo de caso no cenário automobilístico. In: Encontro Nacional de Engenharia de Produção (ENEGEP), 22, 2002. Anais...Curitiba: ABEPRO, 2002.

CUNHA, J. C.; MEDEIROS, J. J. Redes sociais no desenvolvimento de inteligência competitiva. Gestão.Org: Revista Eletrônica de Gestão Organizacional, v. 5, n. 3, set./dez. 2007.

DOCHERTY, M. Primer on "Open Innovation": Principles and Practice. Visions, v. 30, n. 2, p. 13-15, abr. 2006.

FERREIRA, Vicente da Rocha Soares et al. Inovação no setor público federal no Brasil na perspectiva da inovação em serviços. RAI Revista de Administração e Inovação, v. 12, n. 4, p. 99-118, 2014.

FRANCO, M.; BARBEIRA, M. As alianças como estratégia e inteligência competitiva na partilha de conhecimento organizacional. In: Asociación Europea de Dirección y Economía de Empresa, Congreso Nacional, 2, 2008. Anais... Salamanca, 2008. Disponível em: <https://dialnet.unirioja.es/servlet/articulo?codigo=2733594 > Acesso em: 20 ago. 2018

GARCEZ, M. P. et al. Gestão do processo de desenvolvimento de novos produtos: o caso Braskem. Revista de Administração da USP, São Paulo, v. 42, n. 1, p. 19-30, 2005.

GOMES, Elizabeth.; BRAGA, Fabiene. Inteligência competitiva: como transformar informação em um negócio lucrativo. 2. ed. Rio de Janeiro: Campus, 2004. 
HERRING, J. P. Key Intelligence Topics: a process to identify and define intelligence needs. Competitive Intelligence Review, v. 10, n. 2, p. 4-14, 1999.

HUSSEIN, R. D.; FARZANEH, G.; FARHAM, A. Analyzing the impact of competitive intelligence on innovation at scientific research centers In Isfahan science and technology town. Interdisciplinary Journal of Contemporary Research in Business, v. 3, n. 5, set. 2011.

KAHANER, L. The basics of competitive intelligence. In: Competitive Intelligence: How to Gather Analyze and Use Information to Move Your Business to the Top. 1. New York: Simon \& Shuste, 1996.

KUIPERS, S. Crowdsourcing and finding your prosumers. Disponível em: <http://dailycrowdsource.com/2011/12/07/ crowd-leaders/crowdsourcing-andfinding-your-prosumers/>. Acesso em: 12 dez. 2017.

LEMOS, A. D.; PORTO, A. C. Technological forecasting techniques and competitive intelligence: tools for improving the innovation process. Industrial Management \& Data Systems, v. 98, n. 7, p. 330-337, 1998.

LONGANEZI, T.; COUTINHO, P.; BOMTEMPO, J. V. M. Um modelo referencial para a prática da inovação. Journal of Technology Management \& Innovation, v. 3, n. 1, 2008.

LOPES, A. P. V. B. V. et. al. Innovation management: a literature review about the evolution and the different innovation models. In: International Conference on Industrial Engineering and Operations Management, 18, 2012. Anais...Rio de Janeiro: ABEPRO, 2012.

MACHADO, G. R.; SOUZA, E. M. S.; VALVERDE, M. C. S. A análise pest aplicada à prospecção de cenários para o sistema agroindustrial do leite brasileiro. Revista de Administração e Negócios da Amazônia, v. 2, n. 1, jan./abr. 2010.

MARCIAL, E. C.; COSTA, A. J. L. O uso de cenários prospectivos na estratégia empresarial: Evidência especulativa ou Inteligência Competitiva. In: Encontro da Associação Nacional de Pós-Graduação e Pesquisa em Administração (ANPAD), 25, 2001. Anais...Campinas: 2001.

MARCO, S. A. Inteligência competitiva: definições e contextualização. Transinformação, v. 11, n. 2, p. 95-102, maio/ago. 1999.

MENON, A. et al. Antecedents and Consequences of Marketing Strategy Making: A Model and a Test. Journal of Marketing, v. 63, n. 2, p. 18-40, abr. 1999. 
MILLER, S. H. Competitive intelligence - an overview. Competitive

Intelligence Magazine, v. 1, n. 11, 2002. Disponível em: <http://www.ventesmarketing.com/References/Intelligence\%20concurrentielle/Articles/Cl\%20Overv iew.pdf>. Acesso em: 22 set. 2016.

MOHSIN, A. A.; HALIM, H. A.; AHMAD, N. H. Competitive Intelligence Among SMEs: Assessing the Role of Entrepreneurial Attitude Orientation on Innovation Performance. In: International Symposium on Partial Least Squares Path Modeling - The Conference for PLS Users, 2, 2015. Sevilha: 2015.

MONTANHA JUNIOR, I. R. et al. Importância, Definições e Modelos de Inovação. In: CORAL, E.; OGLIARI, A.; ABREU, A. F. (Org.). Gestão Integrada da Inovação: Estratégia, Organização e Desenvolvimento de Produtos. São Paulo: Atlas, 2008. p. 1-13.

MORAIS, F. R.; LUZ, T. R. Tecnologia, Inovação e Competitividade: um estudo de caso sobre redes sociais que utilizam as ferramentas da Web 2.0 para o fortalecimento da produtividade local. In: Simpósio de Gestão da Inovação Tecnológica, 24, 2010. Anais...Vitória: 2010.

NASCIMENTO, L.; LUFT, M. C. M. S.; FREITAS, F. C. H. P. O uso do crowdsourcing como ferramenta de inovação aberta: uma categorização a luz da teoria de redes interorganizacionais. In: Encontro da Encontro da Associação Nacional de Pós-Graduação e Pesquisa em Administração (ANPAD), 36, 2012. Anais...Rio de Janeiro: 2012.

NASCIMENTO, Ademir; HEBER, Florence; LUFT, Maria Conceição. O uso do crowdsourcing como ferramenta de inovação aberta: uma categorização à luz da teoria de redes interorganizacionais. Revista Gestão Organizacional, v. 6, n. 2, 2014.

OLIVEIRA, M. G.; ROZENFELD, H. Alinhando o technology roadmapping (trm) e a gestão de portfólio. In: Seminário technology roadmapping: conceitos, práticas e perspectivas, 2009. São Paulo: 2009.

PANIZZON, M.; OLIVEIRA, R. L. Inteligência competitiva na gestão da inovação de cursos de graduação: um estudo de caso. Revista Inteligência Competitiva, São Paulo, v. 3, n. 3, p. 18-37, jul./set. 2013.

PEREIRA, F. C. M.; CARVALHO, R. B.; JORDÃO, R. V. D. Análise do ciclo da inteligência competitiva em arranjos produtivos locais: estruturação e implantação do bureau de inteligência do apl de software de Belo Horizonte. Revista Inteligência Competitiva, São Paulo, v. 6, n. 1, p. 139-164, jan./mar. 2016.

PHAAL, R.; FARRUKH, C. J. P.; PROBERT, D. R. Technology roadmapping - a planning framework for evolution and revolution. Technological forecasting and social change, v. 71, n. 1-2, p. 5-26, 2004. 
PIZZOL, L. D.; TODESCO, J. L.; TODESCO, B. P. R. Como a web de dados pode apoiar o processo de inteligência competitiva. Perspectivas em Gestão \& Conhecimento, João Pessoa, v. 5, n. especial, p. 87-102, jan. 2016.

PRIPORAS, C. V.; GATSORIS, L.; ZACHARIS, V. Competitive intelligence activity: evidence from Greece. Marketing Intelligence \& Planning, v. 23, n. 7, p. 659-669, 2005.

RODRIGUES, L. C.; et al. As redes de contatos pessoais e as práticas de inteligência competitiva: um estudo sobre a arquitetura de relacionamento em uma empresa de logística. Revista de Negócios, Blumenau, v. 14, n. 4 p. 67 86, out./dez. 2009.

ROUACH, D.; SANTI, P. Competitive Intelligence Adds Value: Five Intelligence Attitudes. European Management Journal, v. 19, n. 5, p. 552-559, out. 2001.

ROZENFELD, H. et al. Gestão de desenvolvimento de produtos: uma referência para a melhoria do processo. São Paulo: Saraiva, 2006.

SANTOS, S. M. M.; SILVA, P. P. P.; SANTOS, J. F. Gabriela Pugliesi: uma análise sobre o marketing de influência na rede social Instagram. In: Congresso de Ciências da Comunicação na Região Nordeste, 18, 2016. Anais...Caruaru: Intercom, 2016.

SCHUMPETER, J. The Theory of Economic Development. An inquiry into Profits, Capital, Credit, Interest, and the Business Cycle. Harvard University Press, Cambridge, 1934.

SILVA, D. O.; BAGNO, R. B; SALERNO, M. S. Modelos para a gestão da inovação: revisão e análise da literatura. Production, v. 24, n. 2, São Paulo, abr./jun. 2014.

TANEV, S.; BAILETTI, T. Competitive intelligence information and innovation in small Canadian firms. European Journal of Marketing, v. 42, n. 7, p. 786-803, 2008.

TIDD, J.; BESSANT, J.; PAVITT, K. Managing innovation: integrating technological, market and organizational change. Journal of Engineering and Technology Management, v. 15, p. 111-113, 1997.

VIDIGAL, F.; MARQUES, L. K. S. Redes Sociais como Fontes de Informação em Inteligência Competitiva: uma abordagem a partir de métodos qualitativos.

Atas - Investigação Qualitativa em Ciências Sociais, v. 3, 2016. 


\title{
THE INTERRELATION BETWEEN THE PROCESS OF COMPETITIVE INTELLIGENCE AND THE MANAGEMENT OF INNOVATION: PROPOSITION OF AN INTEGRATIVE THEORETICAL MODEL
}

\begin{abstract}
Introduction: In face of the inherent uncertainties of innovation process and the increasing instabilities of competitive markets, several methods and techniques have been used to support the new product development and innovation processes. Although the practices of Competitive Intelligence $(\mathrm{Cl})$ are recognized as a strategic process to support decision-making, few papers explore in more detail how the $\mathrm{Cl}$ cycles can be incorporated into the innovation management process in order to strengthen it. Objectives: To propose an integrative theoretical model of the practices of Competitive Intelligence $(\mathrm{Cl})$ with the Stage-Gate ${ }^{\circledR}$ innovation model. Methodology: Literature review. Results: An integrative theoretical model of Stage-Gate® with IC cycles, in which a single cycle is associated with the stages related to the strategic level of innovation process while specific cycles are associated to each of product project separately. Conclusions: The models integration contributes positively to business results by stimulating a value chain of knowledge that allows the continuous reduction of uncertainties, as well a greater knowledge of market and clients, supporting decision making throughout whole innovation process. This highlights the complementarity and advantages of the combined use of approaches discussed here.
\end{abstract}

Descriptors: Innovation Management. Competitive Intelligence. Decision Making. Intelligence Cycle. Conceptual Model.

\section{LA INTERRELACIÓN ENTRE EL PROCESO DE INTELIGENCIA COMPETITIVA Y LA GESTIÓN DE LA INNOVACIÓN: PROPUESTA DE UN MODELO TEÓRICO INTEGRATIVO}

\section{RESUMEN}

Introducción: Frente a las incertidumbres inherentes al proceso de innovación y la inestabilidad de los mercados, cada vez más competitivos, diversos métodos y técnicas son utilizados por las empresas para apoyar el proceso de desarrollo de nuevos productos y la innovación en sí. Aunque las prácticas de Inteligencia Competitiva (IC) sean reconocidas como un proceso estrategico de suporte a la tomada de decisión, pocos trabajos exploran de manera detallada como los ciclos de IC pueden ser incorporados a la gestion de la innovación de forma a tornalo robusto. Objetivos: Proponer un modelo teórico integrador de las prácticas de IC con el modelo de innovación Stage-Gate $\AA^{\circledR}$. Metodología: Revisión de la literatura. Resultados: Modelo teorico integrador del Stage-Gate $\AA$ con los ciclos de IC, en el cual un ciclo unico está asociado a las etapas relacionadas a nivel estratégico del proceso mientras ciclos especificos son asociados a cada uno de los proyectos separadamente. Conclusiones: $\mathrm{La}$ integración de los modelos contribuye positivamente para los resultados empresariales al estimular una cadena de agregación de valor en conocimiento que permite la redución continua de las incertitumbres, así como un mayor conocimiento del mercado y de los clientes, forneciendo apoyo a decisiones durante todo el proceso de

Inf. Inf., Londrina, v. 23, n. 2, p. 427 - 451, maio/ago. 2018.

http://www.uel.br/revistas/informacao/ 
Thaís Silva, Júlia Mudrik, Frederico Vidigal

A inter-relação entre o processo de inteligência competitiva e a gestão da inovação: proposição de um modelo teórico integrativo

decisión y innovación. Se hace importante destacar la complementariedad y las ventajas de la utilización combinada de las abordajes dirigidas aquí.

Descriptores: Innovación de la Gestión. Competitividad de la inteligencia. Toma de decisiones. Inteligencia de Cycle. Conceptual Model.

Recebido: 10.03. 2017

Aceito: 27.02.2018

Inf. Inf., Londrina, v. 23, n. 2, p. 427 - 451, maio/ago. 2018

http://www.uel.br/revistas/informacao/ 